\title{
Design of L-S band broadband power amplifier using microstip lines
}

\author{
Mohamed Ribate ${ }^{1}$, Rachid Mandry ${ }^{2}$, Jamal Zbitou $^{3}$, Larbi El Abdellaoui ${ }^{4}$, Ahmed Errkik ${ }^{5}$, \\ Mohamed Latrach ${ }^{6}$, Ahmed Lakhssassi ${ }^{7}$ \\ 1,2,3,4,5 Mechanical, Energy, Electronics and Telecommunication Laboratory (LMTEE), \\ Faculty of Sciences and Techniques, Hassan 1st University, Morocco \\ ${ }^{6}$ Microwave Group ESEO Angers, France \\ ${ }^{7}$ Quebec University, Canada
}

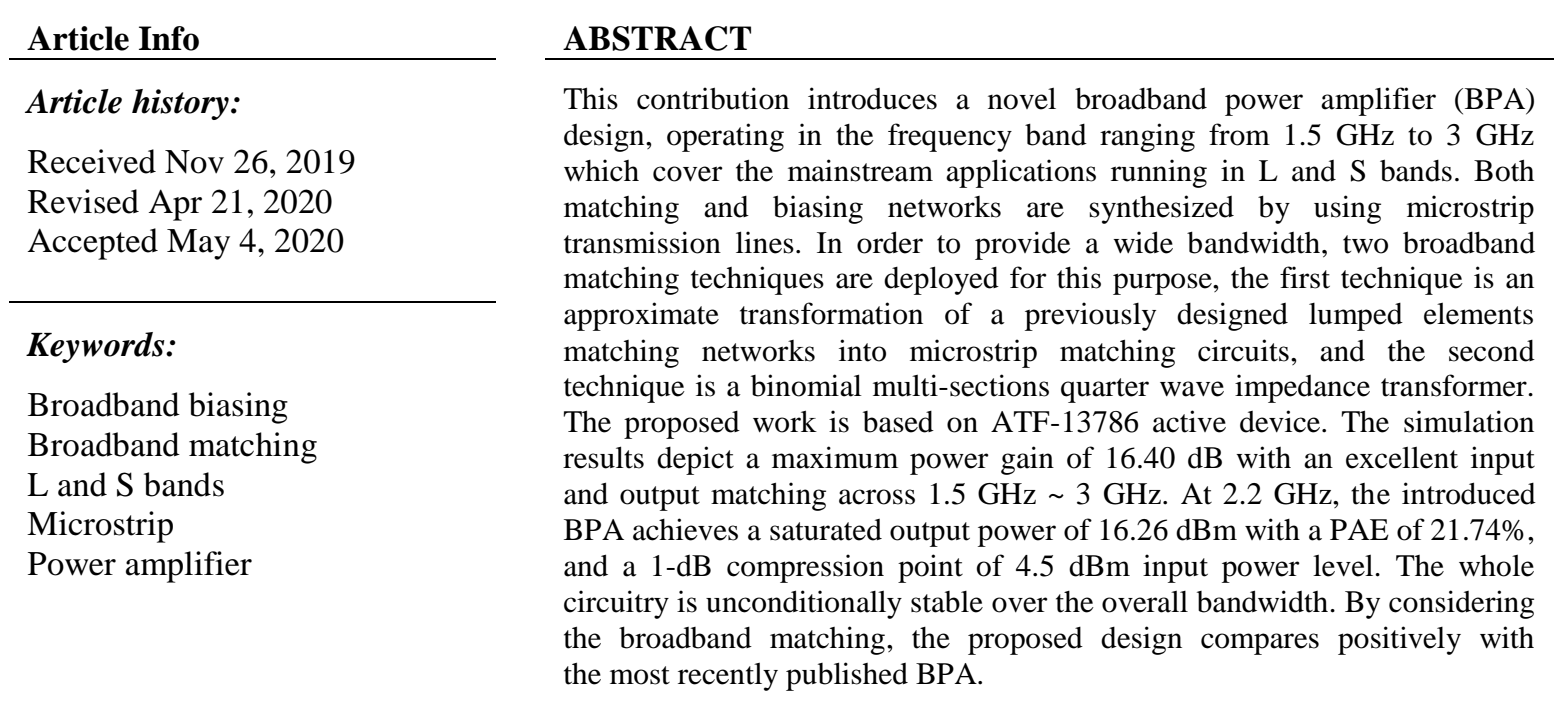

Copyright $@ 2020$ Institute of Advanced Engineering and Science. All rights reserved.

\section{Corresponding Author:}

Mohamed Ribate,

Mechanical, Energy, Electronics and Telecommunication Laboratory (LMEET),

Faculty of Sciences and Techniques, Hassan 1st University,

University Complex, Casablanca Road, Km 3.5, B.O Box 577, Settat, Morocco.

Email: ribate.mohamed@gmail.com

\section{INTRODUCTION}

In today's communication age, the world as we know would be unimaginable without the RF and microwave technology [1]. Nowadays, where telecommunication has turn into a standard, practically, every wireless device has some kind of transceiver, and inherently a power amplifier (PA) permitting it to connect to the mobile cellular networks, Wi-Fi networks, or in a broad sense, to the available wireless networks [2-4]. Basically, PA applications spend a wide range of fields including the traditionally telecommunication, sensing, spectroscopy, navigation, detection of foreign bodies, heating, diagnostics, imaging and treatment applications, and the current trend toward to the Internet of Thing represent just a few examples [5-8].

On the other hand, the cellular mobile communication has reached tremendous advances in terms of technology and innovation as well as commercial accomplishment [9]. However, with the explosive proliferation of the RF and wireless communication standards, driven mostly by the growing demands to transmit an increasing amount of data, besides the growing need of rapid transport of signals in the next generation communication systems as well as the wider signal channels requirement, will lead in a deeply interest in broadband power amplifiers (BPAs) [10-14]. Such devices can supersede multi narrowband and 
single channel PAs, and inherently reduce the hardware research and develop fee due to the incompatibility of the new and previous telecommunication standards.

Ultimately, there are various circuit configurations and topologies deployed to implement broadband power amplifiers including distributed configurations, balanced structures, traveling wave approach and the traditional reactive/resistive matching techniques [15-17]. The latter techniques use resistors in order to synthesis its matching circuits. Although the reactive/resistive schemes are appropriate for bandwidths less than one octave, these techniques show few weak points in terms of VSWR performance and gain flatness as well as necessitate more matching elements. In addition, at high frequencies, the resistors introduce a little effect on the active device owing to the inductive effect of the shunt lines [18-20].

The balanced configuration results in large size, lower PAE and higher noise figure due to the additional loss in the coupler. However, this scheme is good for VSWR performance and gain flatness up to about a two octave bandwidth [21-22]. On the other hand, the traveling-wave and distributed amplifiers have flat gain and wide frequency range, but the main drawbacks for these techniques reside in the moderate small signal gain, high dc power consumption, high noise figure and the large chip size owing to the high number of components used to achieve the same performance as a single stage PA [24-26].

In this work, a novel BPA topology is presented. In order to reach the broadband performance, a hybrid matching techniques are deployed including an approximate transformation of previously designed lumped elements matching circuits into transmission lines matching networks as well as a multi-section binomial transformer. The proposed BPA cover the mainstream wireless communication standards operating in ultra high frequency (UHF) band from $1.5 \mathrm{GHz}$ to $3 \mathrm{GHz}$. This work is based on ATF-13786 active device, and implemented on FR4 substrate.

This paper is organized as follows. Section 2 describes the circuit design including BPA block diagram, the applied matching techniques and the broadband biasing circuit. Section 3 is devoted to the simulation results. Finally, the concluding statements are summarized in section 4.

\section{CIRCUIT DESIGN}

Basically, a power amplifier is a fundamental component, playing a key role in the implementation of many telecommunication systems. However, regardless of its physical implementation as narrowband, broadband, low noise, high efficiency, high power or otherwise, the PA task is to provide a finite power gain in a given frequency band. In other words, increasing the power level of the available signal at its input up to a predefined level at its output in the operating frequency band. From a practical point of view, PA design is usually the result of a trade-off, attempting to fulfil many conflicting requirements. The main concerns are broad bandwidth vs gain flatness, high output power vs low distortion, or linearity vs efficiency.

The PA block diagram is schematically illustrated in Figure 1, where a typical single stage PA consists of a single RF transistor, matched to the source impedance $\left(Z_{S}\right)$ and the load impedance $\left(Z_{L}\right)$ through an input and output matching networks respectively, and DC supplied through a DC bias circuit. We note that both the source and load having the same system characteristic impedance $\mathrm{Z}_{0}$.

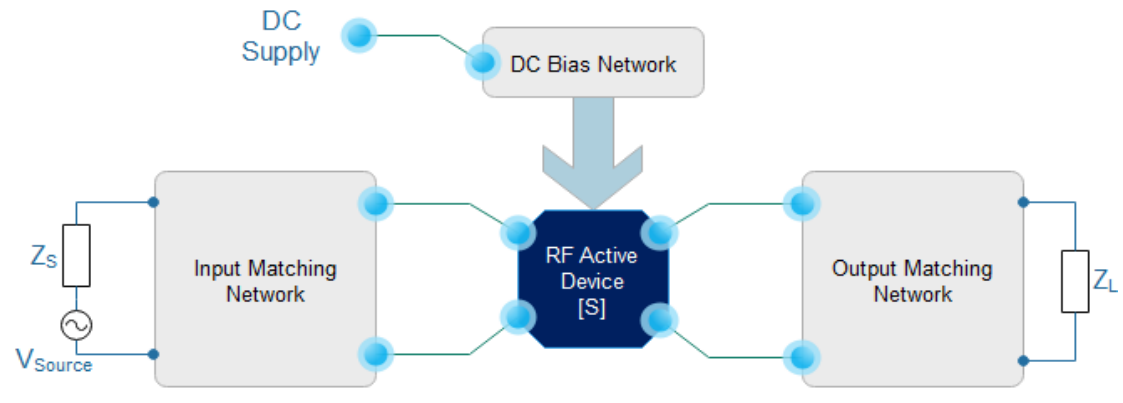

Figure 1. Single stage PA block diagram

\subsection{Broadband matching circuit design}

At high frequencies, the active device is naturally mismatched with the source and load impedances respectively. Consequently, in order to guarantee a maximum transfer of the available RF power from the input port to the active device, from the active device to the output port, or between devices, an appropriate matching networks must be placed at the interface between two mismatched sections, otherwise, a part of the electrical signal transmitted through the PA will be reflected, and this results in a loss of signal. 
However, since the main driven in the BPA design is the need for wider bandwidth, the designed matching circuits must cover a bandwidth as wider as possible. There are various matching techniques that can be deployed for this purpose, but the main factors to be considered in the selection of the right matching technique are, frequency response, bandwidth, ease of implementation and complexity. Consequently, the selection of the appropriate technique might not always be clear. Moreover, the implementation also depends on the technology of fabrication, such as a printed circuit board (PCB), hybrid, or monolithic microwave integrated circuit (MMIC).

Practically, in narrowband and low frequencies applications, the matching networks are generally designed by using lumped elements, because of its flexibility. However, for high frequencies or broadband applications, excepting MMIC technology, it usually very difficult to implement lumped elements on PCB substrate. To solve this problem, transmission lines are used instead of lumped elements when PCB technology is selected.

In this work we use PCB like substrate, as a result, the designed matching circuits are based on transmission lines, in particular on microstrip technology. Moreover, the design method is totally founded on some approximate equations. The first broadband matching technique used in this work is an approximate transformation of the formerly designed lumped elements matching circuits into microstrip matching circuits. This transformation can be done by replacing each resistor, capacitor or inductor with the equivalent transmission line as depicting in Figure 2. The value of the parameters $Z_{1}, Z_{2}, \theta_{1}$ and $\theta_{2}$ can be approximately calculated by using (1) and (2):

$$
\begin{aligned}
& X_{1}=Z_{1} \tan \left(\theta_{1}\right) \approx \omega L \\
& \mathrm{X}_{2}=\frac{Z_{2}}{2 \tan \left(\theta_{2}\right)} \approx \omega C
\end{aligned}
$$

It is worth noting that the microstrip transmission line having the parameters $Z_{1}$ and $\theta_{1}$ represent the series inductor L, while the two parallel-connected microstrip open stubs can be regarded as shunt capacitor C. From a practical stand of point, the field of cross junction illustrated in Figure 2 can be affected when an external circuit is directly connected to the matching network. So to prevent this problem, a transmission line with the same characteristic impedance $Z_{0}$ is added. The second technique deployed in this work is a binomial transformer which is based on multi-sections quarter wave microstrip transmission lines. Figure 3 shows the schematic of this transformer.

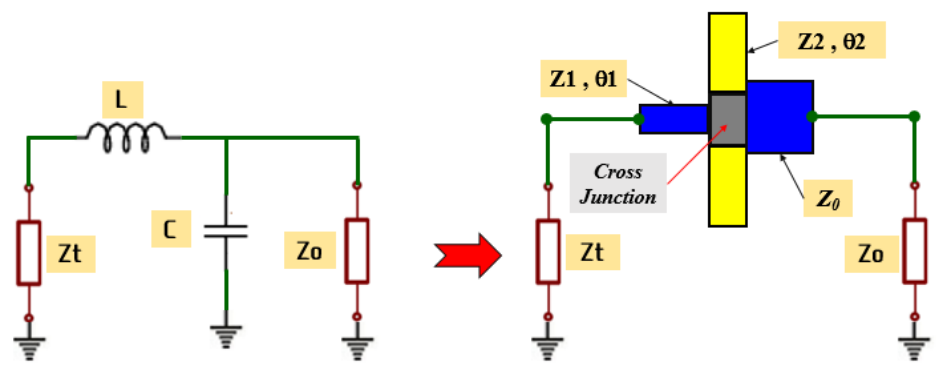

Figure 2. Lumped elements to transmission lines transformation

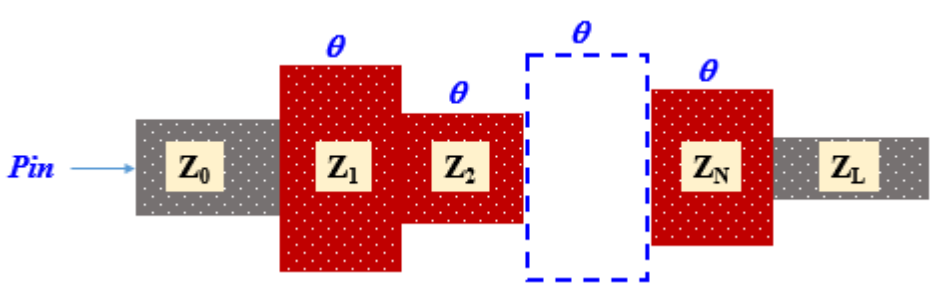

Figure 3. Microstrip multi-sections quarter-wave transformer

The aim of this technique is to transform the load impedance $\mathrm{Z}_{\mathrm{L}}$ to the characteristic impedance $\mathrm{Z}_{0}$ of the input port, by the help of discrete sections of microstrip transmission lines. It is worth noting that 
the transmission lines sections have different characteristic impedance, but the same electrical length $\beta l=\theta$. The latter will be a quarter wavelength at the center of the frequency band $f_{0}$. There are various multi-sections quarter wave transformers, but the widely known of those transformers are Binomial and Chebyshev. In this work, we use the binomial transformer, where its fractal bandwidth is given by the (3):

$$
\frac{\Delta f}{f_{0}}=\frac{2\left(f_{0}-f_{m}\right)}{f_{0}}=2-\frac{\pi}{4} \cos ^{-1}\left|\frac{2 \rho_{m}}{\ln \left({ }^{Z_{L} / Z_{0}}\right)}\right|^{1 / N}
$$

$\mathrm{N}$ is the number of the microstrip transmission lines sections, while $\rho_{\mathrm{m}}$ is the tolerable reflection coefficient in the passband. The transmission lines impedances can be approximately calculated by the help of the (4):

$$
\ln \frac{z_{n+1}}{Z_{n}}=2 \rho_{n}=2^{-N} C_{n}^{N} \ln \frac{z_{L}}{z_{0}}
$$

and

$$
C_{n}^{N}=\frac{N !}{(N-n) ! n !}
$$

where $Z_{n}$ and $Z_{n+1}$ are the impedances of the $n$th and $(n+1)$ th transmission line sections respectively. $\rho n$ is the reflection coefficient at the junction between $Z_{n}$ and $Z_{n+1}$, and $C_{n}^{N}$ are the binomial coefficient. For example, in the case of two-section transmission lines, by using (4) and (5), the impedances of $Z_{1}$ and $Z_{2}$ are given by:

$$
\begin{aligned}
& Z_{1}=Z_{L}^{1 / 4} Z_{0}^{3 / 4} \\
& Z_{2}=Z_{L}^{3 / 4} Z_{0}^{1 / 4}
\end{aligned}
$$

\subsection{Broadband biasing circuit design}

Biasing circuits are essential parts of BPA design. Its main job is to provide the efficient excitation in terms of current and voltage for the active device. The biasing parameters can vary with the active device technology, but the design principles for biasing techniques are ultimately the same. There are many biasing schemes for BPA deployed in practice. It's worth noting that the biasing networks could be integrated in the matching circuitry or they can be stand-alone.

Typically, a generic RF biasing circuit as depicted in Figure 4(a), consists of a DC block and RF choke. The aim of DC block is to present a short-circuit at the operational bandwidth, while the role of RF choke is to prevent microwave signals from leaking into the biasing network. Consequently, the RF choke is synthesized in a such a way that behaves as a very high impedance in the operating frequency band. In this work, the biasing circuit is an integral part of the matching network, and it is synthesized using microstrip transmission lines. The capacitor value is selected in a such a way that is very high. The active device in this work is supplied with a single supply voltage of $+2.5 \mathrm{~V}$. By using the principles mentioned above, the proposed BPA layout is shown in Figure 5.

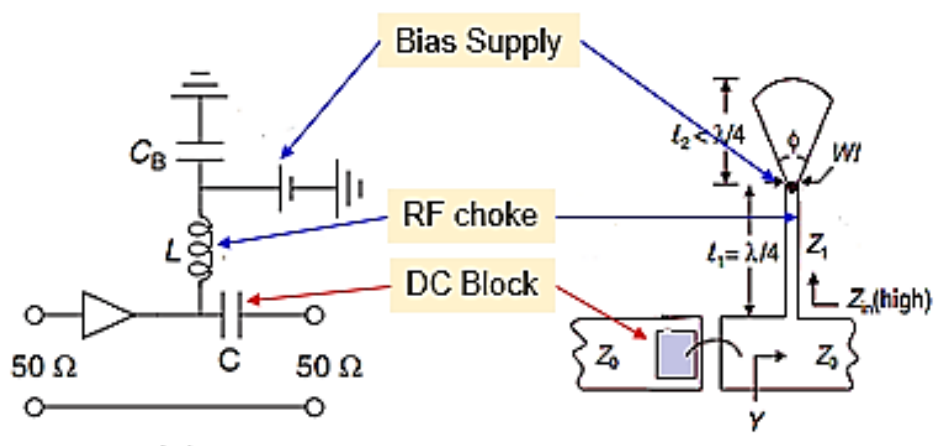

(a)

(b)

Figure 4. (a) Generic RF biasing configuration, (b) Broadband biasing configuration 


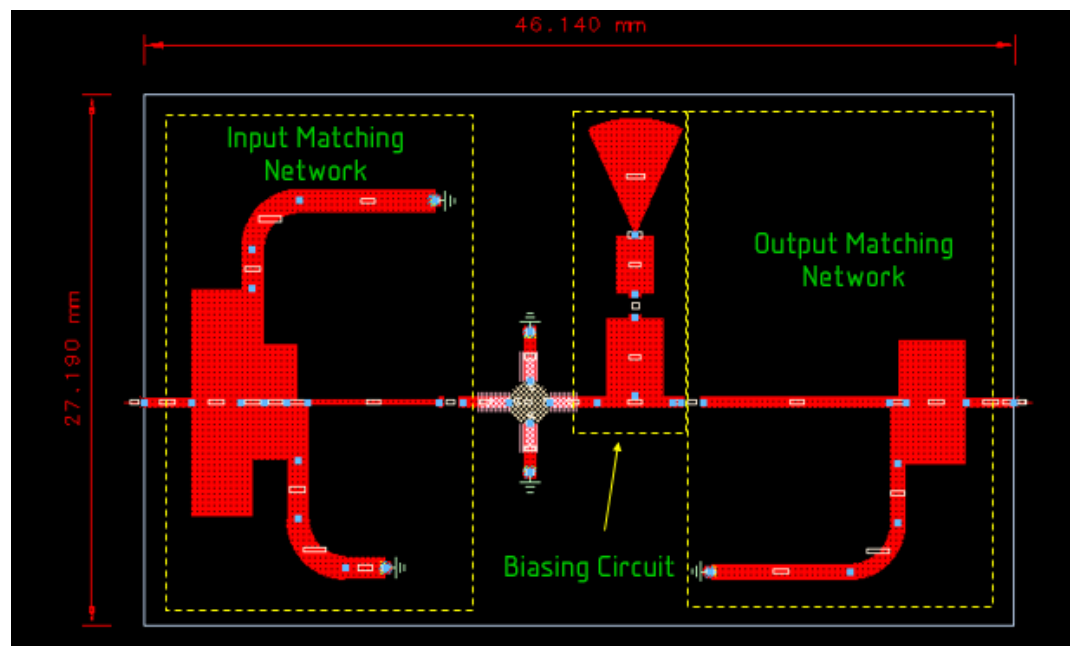

Figure 5. The proposed broadband power amplifier layout

\section{RESULTS AND ANALYSIS}

\subsection{Small-signal analysis}

At high frequencies, it's very complicated to characterize a two port device by using the immittance parameters, because the measurement of the current and voltage under open and short circuits is very difficult. Consequently, in order to solve this problem, the characterization of RF power amplifier is done by using the scattering parameters, which describe the behavior of a given two port circuit in terms of incident and reflected power waves at the circuit terminals. The source and load terminations deployed in this works both having the same impedance of $50 \Omega$. Therefore, in this work, the effectiveness of the designed matching networks is evaluated by means of S-parameters simulation using ADS software.

Figure 6 shows the small-signal scattering parameters of the proposed BPA. As reported in Figure $6(\mathrm{a})$, the input return loss $\left[\mathrm{S}_{11}\right]$ is strictly under $-10 \mathrm{~dB}$, while the output return loss $\left[\mathrm{S}_{22}\right]$ is purely under $-14 \mathrm{~dB}$ over the frequency band ranging from $1.5 \mathrm{GHz}$ to $3 \mathrm{GHz}$. However, from Figure 6(b), the small-signal power gain $\left[\mathrm{S}_{21}\right]$ reaches a maximum value of $16.40 \mathrm{~dB}$, while the minimum value of this parameters is $10 \mathrm{~dB}$. The revers transmission coefficient $\left[\mathrm{S}_{12}\right]$ also achieves a maximum value of $-18.53 \mathrm{~dB}$ over the operating bandwidth. From the obtained results, the microstrip biasing network as well as the deployed broadband matching techniques show excellent results in terms of reflexion coefficient, revers transmission and small signal gain over a wide bandwidth from $1.5 \mathrm{GHz}$ to $3 \mathrm{GHz}$.

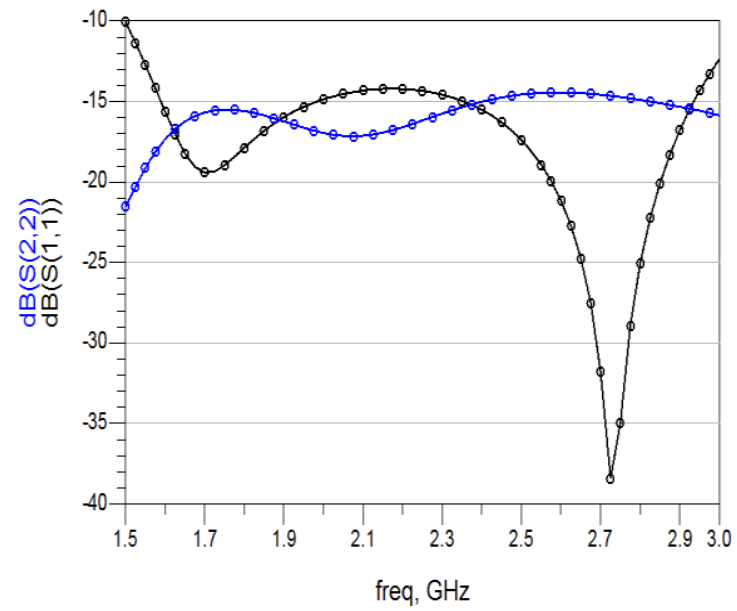

(a)

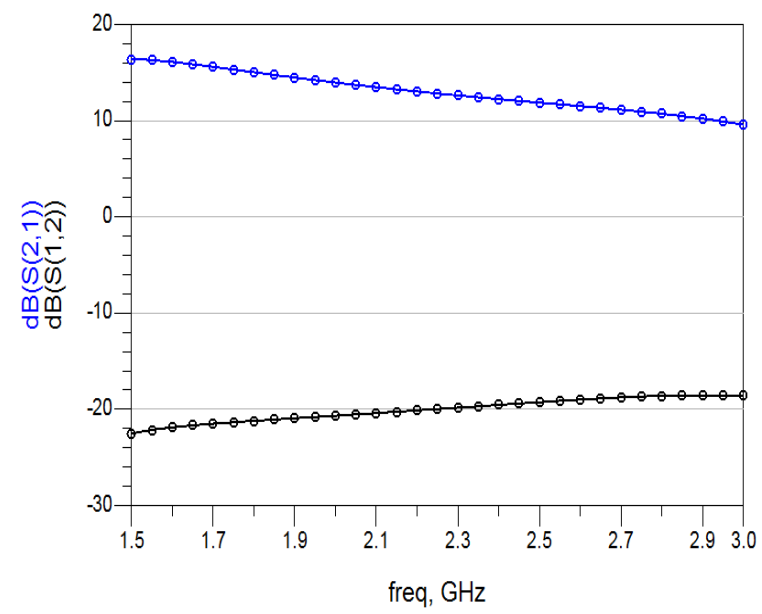

(b)

Figure 6. Small signal scattering parameters, (a) input return loss $\left[\mathrm{S}_{11}\right]$ and output return loss $\left[\mathrm{S}_{22}\right]$,

(b) small-signal gain $\left[\mathrm{S}_{21}\right]$ and revers transmission coefficient $\left[\mathrm{S}_{12}\right]$ 


\subsection{Stability analysis}

Basically, every power amplifier must fulfill the unconditionally stability requirement in the operating frequency, if not, the unavoidable parasitic effects are enough to introduce oscillations and turn it into an oscillator. Two stability factors are commonly used for this purpose, Bodway $\left(\mathrm{B}_{1}\right)$ and Rollet $(\mathrm{k})$ stability factors which are defined by the (8) and (9):

$$
\begin{aligned}
& B_{1}=1+\left|S_{11}\right|^{2}+\left|S_{22}\right|^{2}-|\Delta|^{2}>0 \\
& k=\frac{1+|\Delta|^{2}-\left|S_{11}\right|^{2}-\left|S_{22}\right|^{2}}{2\left|S_{12} S_{21}\right|}>1
\end{aligned}
$$

In addition to the (8) and (9), the unconditionally stability is realized only if the following conditions are accomplished simultaneously: $S_{22}<1, B 1>0$ and $\mathrm{K}>1$. Figure 7 shows the curves of the Rollet and Bodway stability factors versus frequency, where the minimum value achieved by $\mathrm{k}$ and $\mathrm{B}_{1}$ is 1.7 and 0.7 respectively over the frequency band ranging from $1.5 \mathrm{GHz}$ to $3 \mathrm{GHz}$. According Figure 8, the unconditionally stability requirement of the propose BPA is fulfilled. That means that any source or load impedances can be connected without risking to have oscillations.

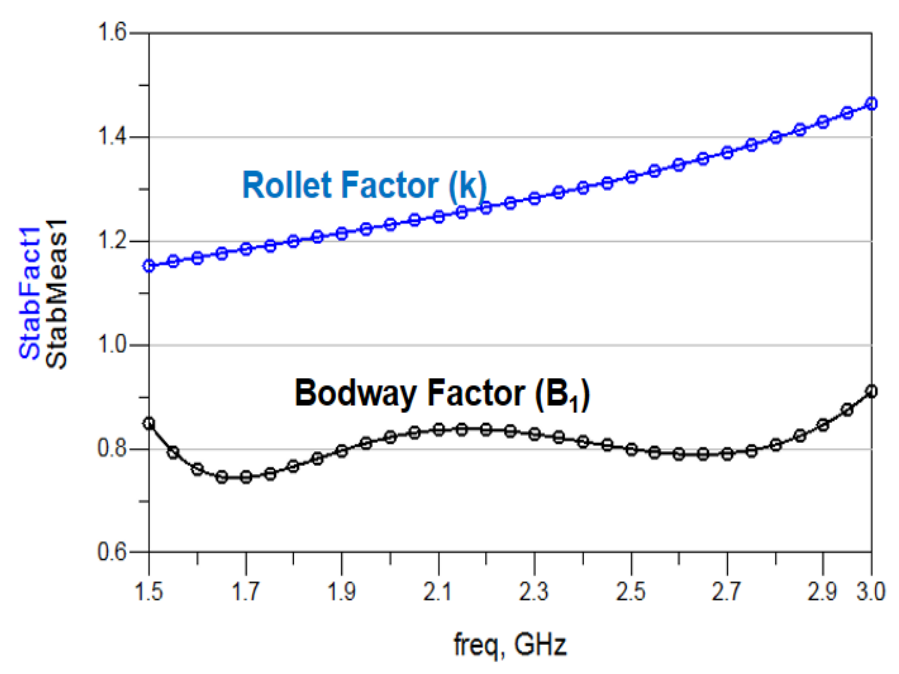

Figure 7. Rollet and bodway stability factors curves versus frequency

\subsection{Large-signal performances}

In this sub-section, the output power, 1-dB compression point and the power added efficiency (PAE) are presented. The large-signal performance of the proposed BPA has been simulated at $2.2 \mathrm{GHz}$, under $50 \Omega$ input and output impedances. Figure 8 shows the simulated output power and the 1-dB compression point of the proposed BPA.

From Figure 8, the proposed BPA reaches a maximum output power of $16.26 \mathrm{dBm}$, this leading of $42.26 \mathrm{~mW}$. However, as the input power continues to increase, we ultimately reach a point where no further output power increases occur for an input power increase. This point named 1-dB compression point in which the BPA goes into compression and becomes saturated. As depicted in Figure 8, the 1-dB compression point of the proposed BPA is $4.5 \mathrm{dBm}$ of input power level.

On the other hand, from a practical point of view, a power amplifier can be regarded as a device that transform the DC power from supplies into RF power in the operating frequency band. The effectiveness of this conversion is eventually measured by means of power added efficiency (PAE), which is defined as the ration between the added power and the supplied DC power. The simulated PAE of the introduced BPA is shown in Figure 9, in which, the proposed work achieves a maximum PAE of $21.74 \%$ at $2.2 \mathrm{GHz}$. With the aim to evaluate the deployed broadband matching techniques, a comparison with the similar contemporary state-of-the-art works is performed. From Table 1, to author's best acknowledge, the proposed work presents competitive performances in terms of gain, broadband matching, PAE and output power over a broad bandwidth ranging from $1.5 \mathrm{GHz}$ to $3 \mathrm{GHz}$. 




Figure 8. Output power and the 1-dB compression point

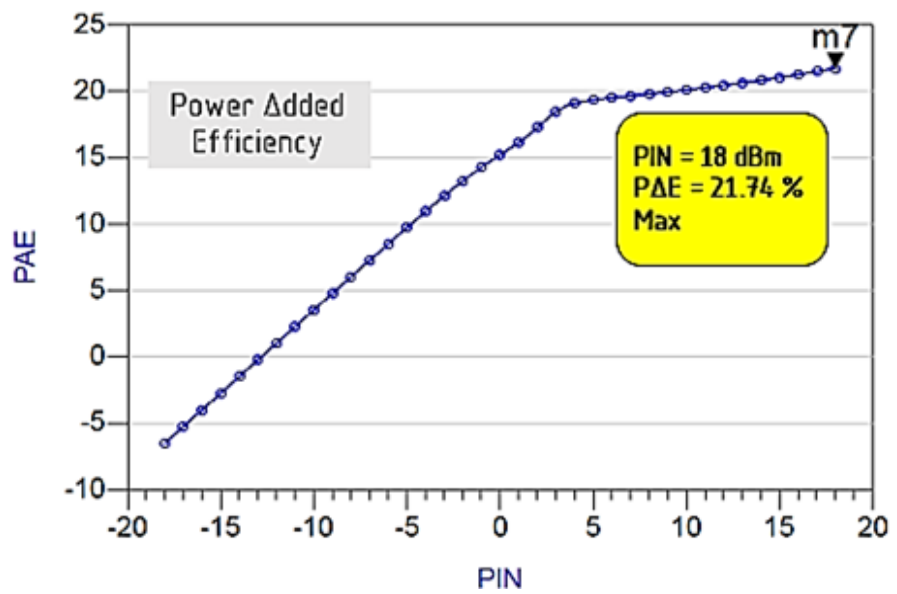

Figure 9. Power added efficiency (PAE)

Table 1. Comparison between some recently published BPA

\begin{tabular}{cccccccc}
\hline PAs & $\begin{array}{c}\text { Freq } \\
{[\mathrm{GHz}]}\end{array}$ & $\begin{array}{c}\mathrm{S}_{11} \\
{[\mathrm{~dB}]}\end{array}$ & $\begin{array}{c}\mathrm{S}_{22} \\
{[\mathrm{~dB}]}\end{array}$ & $\begin{array}{c}\text { Gain } \\
{[\mathrm{dB}]}\end{array}$ & $\begin{array}{c}\text { Psat } \\
{[\mathrm{dBm}]}\end{array}$ & $\begin{array}{c}\text { PAE } \\
{[\%]}\end{array}$ & $\begin{array}{c}\text { Supply } \\
{[\mathrm{V}]}\end{array}$ \\
\hline$[22] 2015$ & $1.9-2.7$ & - & - & 11 & 28.1 & 13.7 & 2.5 \\
{$[21] 2016$} & $1.8-2.8$ & $-12 \mid-25$ & $-9 \mid-19$ & 28 & 25 & 6.1 & 5 \\
{$[4] 2017$} & $1-4$ & $-9 \mid-24$ & $-8 \mid-18$ & 12.15 & 14.8 & 20 & 3.5 \\
{$[10] 2018$} & $1.1-3$ & $-10 \mid-35$ & $-10 \mid-25$ & 14.9 & 17.14 & 14.9 & 3 \\
{$[23] 2018$} & $1.75-2.15$ & $-11 \mid-22$ & $-13 \mid-19$ & - & 8 & 11.7 & - \\
{$[9] 2019$} & $1.5-2.6$ & - & - & 9.3 & 45 & $-2.9 \mid-5.6$ \\
This Work & $1.5-3$ & $-10 \mid-38$ & $-14 \mid-24$ & 16.40 & 16.76 & 21.74 & 2.5 \\
\hline
\end{tabular}

\section{CONCLUSION}

In this paper, a broadband power amplifier operating in the frequency band ranging from $1.5 \mathrm{GHz}$ to $3 \mathrm{GHz}$ is presented. The active device used in this work is ATF-13786. The proposed BPA cover the mainstream applications running in L and S bands. With the deployed broadband matching techniques as well as the integral microstrip biasing network, excellent input and output matching is achieved under unconditionally stability over a broad bandwidth. The large signal simulation indicates good output power and PAE with a 1-dB compression point of $4.5 \mathrm{dBm}$ input power level. A power amplifier with those features is more appropriate for many $\mathrm{L}$ and $\mathrm{S}$ applications including cellular communication standards, medical applications (radiometry, hyperthermia, imaging...), navigation, microwave heating and many other applications. 


\section{REFERENCES}

[1] M. Ribate, et al., "1.25 GHz - 3.3 GHz Broadband Solid State Power Amplifier for L and S Bands Applications," International Journal of Electrical and Computer Engineering (IJECE), vol. 9, no. 5, pp. 3633-3641, Oct. 2019.

[2] M. Bozanic and S. Sinha, "Millimeter-Wave Low Noise Amplifiers," Springer, Switzerland, 2018.

[3] M. Bozanic and S. Sinha, "Power Amplifiers for the S-, C-, X- and ku-bands," Springer, Switzerland, 2016.

[4] M. Ribate, et al., "GaAs FET broadband power amplifier for L and S bands applications," in Proceedings of the $2^{\text {nd }}$ International Conference on Computing and Wireless Communication Systems, no. 42, pp. 1-5, Nov. 2017.

[5] IEEE, "IEEE Standard Letter Designations for Radar-Frequency Bands," IEEE Std., 521-1984, pp. 1-8, 1984.

[6] P. B. Khannur, et al., "A Universal UHF RFID Reader IC in 0.18- $\mu \mathrm{m}$ CMOS Technology," IEEE Journal of Solid State Circuits, vol. 43, no. 5, pp. 1146-1155, May 2008.

[7] A. Rosen, et al., "Applications of RF/microwaves in medicine," IEEE Transactions on Microwave Theory and Techniques, vol. 50, no. 3, pp. 963-974, Aug. 2002.

[8] E. C. Fear, et al., "Confocal microwave imaging for breast cancer detection: localization of tumors in three dimensions," IEEE Transactions on Biomedical Engineering, vol. 49, no. 8, pp. 812-822, Aug. 2002.

[9] D. Gan and W. Shi, "Design of a Broadband Doherty Power Amplifier Based on Hybrid Continuous Mode," IEEE Access, vol. 7, pp. 86194-86204, 2019.

[10] M. Ribate, et al., "Broadband GaAs FET Power Amplifier for L and S Bands Applications," International Journal of Intelligent Engineering and Systems, vol. 11, no. 5, pp. 96-104, 2018.

[11] J. Fang, et al., "3.5 GHz WiMAX GaN Doherty power amplifier with second harmonic tuning," Microwave and Optical Technology Letters, vol. 54, no. 11, pp. 2601-2605, Aug. 2012.

[12] D. Ye, et al., "Tradeoff Design of Broadband Power Amplifier in Doherty Configuration Utilizing a Novel Coupled-Line Coupler," Progress in Electromagnetics Research C, vol. 48, pp. 11-19, 2014.

[13] G. Watkins and S. Wang, "The impact of power amplifier turn-on characteristics in cognitive radio networks," Microwave Journal, vol. 3, pp. 86-92, Mar. 2014.

[14] F. H. Raab, et al., "RF and microwave power amplifier and transmitter technologies - part 1," High Frequency Electronics, pp. 22-36, May 2003

[15] A. Eroglu, "Introduction to RF Power Amplifier Design and Simulation," CRC Press, 2016.

[16] M. K. Kazimierczuk, "RF Power Amplifiers," $2^{\text {nd }}$ ed., John Wiley \& Sons, 2015.

[17] P. Colantonio, et al., "High Efficiency RF and Microwave Solid State Power Amplifiers," UK, John Wiley \& Sons Ltd, 2009.

[18] I. J. Bahl, "Fundamentals of RF and Microwave Transistor Amplifiers," John Wiley \& Sons, 2009.

[19] A. Alizadeh, et al., "Design of a 2-12-GHz Bidirectional Distributed Amplifier in a 0.18- $\mu \mathrm{m}$ CMOS Technology," IEEE Transactions on Microwave Theory and Techniques, vol. 67, no. 2, pp. 754-764, 2019.

[20] T. Ma and F. Hu, "A Wideband Flat Gain Low Noise Amplifier Using Active Inductor for Input Matching," IEEE Transactions on Circuits and Systems II: Express Briefs, vol. 66, no. 6, pp. 904-908, 2019.

[21] C. Q. Chen, et al., "A 1.8-2.8 GHz Highly Linear Broadband Power Amplifier for LTE-A Application,” Progress in Electromagnetics Research C, vol. 66, pp. 47-54, 2016.

[22] B. Francois and P. Reynaert, "Highly linear fully integrated wideband RF PA for LTE advanced in 180-nm SOI," IEEE Transactions on Microwave Theory and Techniques, vol. 63, no. 2, pp. 649-658, Feb 2015.

[23] A. Rachakh, et al., "A Novel Configuration of a Microstrip Microwave Wideband Power Amplifier for Wireless Application," TELKOMNIKA Telecommunication Computing Electronics and Control, vol. 16, no. 1, pp. 224-231, Feb. 2018

[24] M. Ribate, et al., "GaAs Solid State Broadband Power Amplifier for L and S Bands Applications," Handbook of Research on Recent Developments in Electrical and Mechanical Engineering, IGI Global, Chapter 5, pp. 156-190, 2020.

[25] J. Moreno Rubio, et.al., "Design of an $87 \%$ Fractional Bandwidth Doherty Power Amplifier Supported by a Simplified Bandwidth Estimation Method," IEEE Transactions on Microwave Theory and Techniques, vol. 66, no. 3, pp. 1319-1327, Mar. 2018

[26] W.A. Malik, et al. "A $1.8 \mathrm{GHz}$ wideband high power amplifier with flat gain response by exploiting microstrip high-pass-filter," Microwave and Optical Technology Letters, vol. 60, no. 3, pp. 765-769, Mar. 2018

\section{BIOGRAPHIES OF AUTHORS}

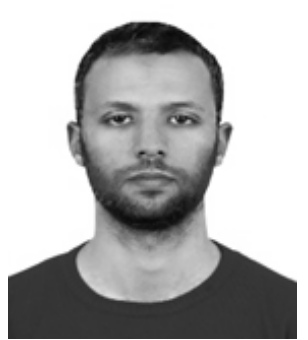

Mohamed Ribate received B.S degree and Master Eng. in Networking and Telecommunication engineering from Hassan $1^{\text {st }}$ University, Settat, Morocco in 2013 and 2015 respectively. He is currently working towards his Ph.D. in Microwave and RF Electronics at FST of Settat, University Hassan 1st, Morocco since November 2015. His research involves design and implementation of Broadband Power Amplifiers using Microstrip Technology. 

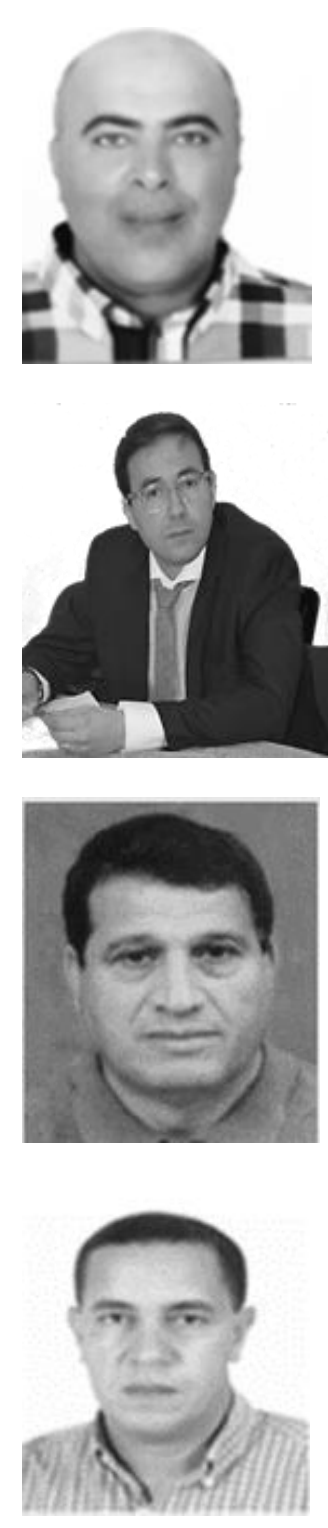

Ahmed Errkik was born in July 1960 in Morocco. He received the Ph.D. degree in physics from the University of Technology Compiegne (UTC), France. He is currently an associate Professor of physics in FST University Hassan 1st, Settat, Morocco. He is involved in the design of hybrid, monolithic active and passive microwave electronic circuits.



Mohamed Latrach was born in Douar Ksiba, Sless, Morocco, in 1958. He received the Ph.D. degree in electronics from the University of Limoges, Limoges, France, in 1990. He is currently a Professor of microwave engineering with the Ecole Suprieure d'Electronique de l'Ouest (ESEO), Angers, France, where his research involves RF and microwaves. His field of interest is the design of hybrid, monolithic active, and passive microwave circuits, metamaterials, LH materials, antennas and their applications in wireless Communications.

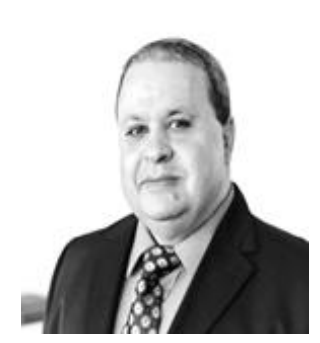

Ahmed Lakhssassi was received the B.Eng. and M.Sc. in electrical engineering from University of Quebec à Trois-Rivières, Quebec, Canada in 1988 and 1990 respectively. He also received the Ph. D in Energy and Material sciences in 1995 from INRS-Energie et Matériaux, Quebec, Canada. His research interest is the fields of bio-heat thermal modeling. Also, his research interest is in Design of fully automated tool for porting analog and mixed signal circuits within different technology nodes. 